\title{
ADMISSIBLE ORDERS OF JORDAN LOOPS
}

\author{
MICHAEL K. KINYON, KYLE PULA, AND PETR VOJTĚCHOVSKÝ
}

\begin{abstract}
A commutative loop is Jordan if it satisfies the identity $x^{2}(y x)=$ $\left(x^{2} y\right) x$. Using an amalgam construction and its generalizations, we prove that a nonassociative Jordan loop of order $n$ exists if and only if $n \geq 6$ and $n \neq 9$. We also consider whether powers of elements in Jordan loops are well-defined, and we construct an infinite family of finite simple nonassociative Jordan loops.
\end{abstract}

\section{INTRODUCTION}

A magma $(Q, \cdot)$ is a quasigroup if, for each $a, b \in Q$, the equations $a x=b$, $y a=b$ have unique solutions $x, y \in Q$. A loop is a quasigroup with a neutral element. Standard references on quasigroup and loop theory are [2, 10].

Most literature on loop theory focuses on varieties of loops satisfying some nearassociativity conditions with strong structural consequences. For instance, Moufang loops defined by the identity $x(y(x z))=((x y) x) z$ are diassociative (every two elements generate a subgroup), and thus power associative (every element generates a subgroup). In contrast, we will be dealing with Jordan loops whose properties are rather weak indeed, and which consequently did not receive much attention yet.

A commutative loop is said to be Jordan if it satisfies the Jordan identity

$$
x^{2}(y x)=\left(x^{2} y\right) x .
$$

This is the same identity used in the definition of Jordan algebras 8 .

While studying Jordan loop rings, Goodaire and Keeping [6] asked for which integers $n$ there exists a nonassociative (that is, not associative) Jordan loop of order $n$. We answer their question here:

Theorem 1.1. A nonasssociative Jordan loop of order $n$ exists if and only if $n \geq 6$ and $n \neq 9$.

By the nature of the problem, this paper consists mostly of constructions, many of which have a combinatorial flavor.

It is not difficult to find nonassociative Jordan loops of even order $n \geq 6$. This part of the problem was already solved by Goodaire and Keeping in [6]. In $\$ 2$, we construct the same loops as in [6], but using a visual argument. The core of our construction is the classical correspondence between idempotent quasigroups and loops of exponent 2 (Proposition 2.2).

The observation that an idempotent quasigroup is a union of singleton subquasigroups suggests that the aforementioned correspondence can be generalized. This is the idea behind an amalgam construction of Foguel [5]: idempotent quasigroups are generalized to quasigroups which are unions of subquasigroups all of the same

2000 Mathematics Subject Classification. 20N05.

Key words and phrases. Jordan loop, Jordan quasigroup, amalgam construction for quasigroups and loops, well-defined powers, nonassociative loop, order of a loop. 
order. In 43 , we use this idea to build Jordan loops of all odd orders except those of the form $2^{m}+1$.

The amalgam construction can be generalized even further, this time by allowing quasigroups that are unions of subquasigroups which are not necessarily of the same order. In 4 we use this further generalization to construct Jordan loops of the troublesome orders $2^{m}+1$ for $m>3$.

In $\$ 5$ we ask if powers $x^{k}$ in Jordan loops are well-defined, where by well-defined we mean that $x^{k}$ has the same value regardless of how it is parenthesized. All orders $0 \leq k \leq 5$ are well-defined (Lemma 5.2), and this is enough to show that the only Jordan loop of order 5 is the cyclic group (Corollary 5.4). Up to relying on a computer search for the case $n=9$, this will complete the proof of Theorem 1.1. A complete human proof of that case will appear elsewhere [1].

We also show that for every composite integer $m n$ where $m>1$ and $n \geq 3$ is odd, there exists a 1-generated Jordan loop with an element $c$ such that all powers $c^{k}$ are well-defined for $0 \leq k<m n$, but for which $c^{m n}$ is not well-defined (Theorem 5.5). The prime and power of 2 cases are more delicate and still open.

Finally, in $\sqrt{6}$, we construct an infinite family of nonassociative simple Jordan loops of order $2^{m}-1$.

We are pleased to acknowledge the assistance of the finite model builder Mace4 developed by McCune 9 . We found many of our constructions by examining small Jordan loops built by Mace4. In addition, in this paper, we rely on our Mace4 findings to complete the obstinate case $n=9$ of Theorem 1.1

\section{THE EVEN ORDER CASE}

The even order case is easy to handle, partly thanks to the following observation.

Lemma 2.1. Every commutative loop of exponent 2 is a Jordan loop.

Proof. Since each $x^{2}=1$, the Jordan identity (J) holds trivially.

Our construction begins with the following classical correspondence, which we need only in the commutative case.

Proposition 2.2. There is a one-to-one correspondence between commutative loops of exponent 2 and order $n>1$, and commutative idempotent quasigroups of order $n-1$.

Proof. Given a commutative idempotent quasigroup on $X=\left\{x_{2}, \ldots, x_{n}\right\}$, introduce a new element 1 , let $1 \cdot x=x \cdot 1=x$ for every $x \in X \cup\{1\}$, set $x_{i} \cdot x_{i}=1$, and leave $x_{i} \cdot x_{j}$ intact for $i \neq j$. Conversely, given a nontrivial commutative loop of exponent 2 on $\left\{1, x_{2}, \ldots, x_{n}\right\}$, remove 1 , set $x_{i} x_{i}=x_{i}$, and leave $x_{i} \cdot x_{j}$ intact for $i \neq j$.

Perhaps the most prominent application of the correspondence of Proposition 2.2 is that between Steiner quasigroups (or Steiner triple systems) and Steiner loops, i.e., commutative loops satisfying $x(x y)=y$ [4]. We note in passing that it is not trivial to prove that a Steiner loop of order $n$ exists if and only if $n$ is congruent to 2 or 4 modulo 6 [7.

We now use Proposition 2.2 to construct the loops of exponent 2 given by Goodaire and Keeping in [6]. We give a visual argument, leaving to the reader the arithmetic details (easily extracted from [6]). First we show that this construction can handle no more than the even order case. 
Lemma 2.3. Let $Q$ be a finite commutative quasigroup. Then $|Q|$ is odd if and only if the squaring map $x \mapsto x^{2}$ is a bijection.

Proof. Let $M$ be a multiplication table for $Q$ arranged so that columns and rows are labeled in the same order. For each $a \in Q$, let $d(a)$ denote the number of times $a$ appears on the main diagonal of $M$. By commutativity, each $a$ occurs the same number of times above the main diagonal of $M$ as it does below the main diagonal. Since there are $|Q|$ occurrences of $a$ in $M$, it follows that $|Q|-d(a)$ is even.

Now if $x \mapsto x^{2}$ is not bijective, then by the finiteness of $Q$, the map is not surjective. Thus for some $a \in Q, d(a)=0$, and so $|Q|$ is even.

Conversely, if $x \mapsto x^{2}$ is a bijection, then for each $a \in Q, d(a)=1$. Therefore $|Q|$ is odd.

Corollary 2.4. Let $Q$ be a finite commutative loop. If there exists $a \in Q, a \neq 1$ such that $a^{2}=1$, then $|Q|$ is even. In particular, every nontrivial, finite, commutative loop of exponent 2 has even order.

Corollary 2.5. Every finite commutative idempotent quasigroup has odd order.

Lemma 2.6. For each odd $n>0$, there exists a commutative idempotent quasigroup of order $n$.

Proof. We will construct a multiplication table for such a quasigroup defined on the set $S=\{1, \ldots, n\}, n$ odd. Place $1, \ldots, n$ on the diagonal, in this order, that is, define $i \cdot i=i$ for all $i \in S$. Then fill out the rest of the table so that all antidiagonals (with wrap around) are constant. This works since $n$ is odd. The resulting quasigroup is clearly commutative and idempotent.

Alternatively, if we let $S=\mathbb{Z}_{n}$ and define the quasigroup operation $*$ by $a * b=$ $(a+b) / 2 \bmod n$, we arrive at the same idempotent quasigroup described above.

Recall that a loop is left alternative if it satisfies $x(x y)=(x x) y$.

Proposition 2.7. Let $n \geq 6$ be even. Then there is a commutative loop of exponent 2 and order $n$ that is not left alternative, hence not associative.

Proof. Let $n \geq 6$ be even. Construct the commutative idempotent quasigroup $Q$ on $\{2, \ldots, n\}$ as in the proof of Lemma 2.6. Let $L$ be the commutative loop of exponent 2 obtained from $Q$ by the correspondence of Proposition 2.2. We claim that $L$ is not left alternative.

Let $k=(n+2) / 2$ be the average of $2, \ldots, n$. Hence $k$ is on the main antidiagonal in the canonical multiplication table of $Q$. Then $k \cdot(k+1)=2$ and $k \cdot(k+2)=k+1$ (here we use the fact that $k+2 \leq n$, or, equivalently, $n \geq 6)$. Thus $(k \cdot k) \cdot(k+1)=$ $1 \cdot(k+1)=k+1$, while $k \cdot(k \cdot(k+1))=k \cdot 2 \neq k+1$.

It is well-known that every loop of order less than 5 is associative. We have therefore established the even case of Theorem 1.1.

\section{The amalgam COnStruction}

Let $(G, \circ)$ be a quasigroup, and let $S$ be a nonempty set. Let $\nabla=\left\{\nabla_{g, h} \mid g, h \in\right.$ $G\}$ be a family of binary operations on $S$ such that each $Q_{g, h}=\left(S, \nabla_{g, h}\right)$ is a quasigroup, and let $\mathcal{Q}=\left\{Q_{g, h} \mid g, h \in G\right\}$ denote the family of all such quasigroups. The quasigroup amalgam $\mathcal{A}(G, \mathcal{Q})$ is the quasigroup defined on $S \times G$ by

$$
(s, g)(t, h)=\left(s \nabla_{g, h} t, g \circ h\right) .
$$


Since it is always clear which multiplication $\nabla_{g, h}$ is supposed to be used in (3.1), we suppress the name of the binary operation in $Q_{g, h}$ and write $s t$ instead of $s \nabla_{g, h} t$.

The term "amalgam" was suggested by Foguel [5]. In fact, this same construction was first introduced by Bruck [1, §10], who called it an "extension" of $G$ by $S$. (However, this is not necessarily an extension in the sense that the term is generally used in algebra.) An extensive list of applications of the quasigroup amalgam can be found in 3 , pp. 36-43].

With the appropriate labeling of rows and columns, we can depict the multiplication table of $\mathcal{A}(G, \mathcal{Q})$ as

\begin{tabular}{c||c|c|c|c}
$\mathcal{A}$ & $g$ & $h$ & $k$ & $\ldots$ \\
\hline \hline$g$ & $\left(Q_{g, g}, g \circ g\right)$ & $\left(Q_{g, h}, g \circ h\right)$ & $\left(Q_{g, k}, g \circ k\right)$ & \\
\hline$h$ & $\left(Q_{h, g}, h \circ g\right)$ & $\left(Q_{h, h}, h \circ h\right)$ & $\left(Q_{h, k}, h \circ k\right)$ & \\
\hline$k$ & $\left(Q_{k, g}, k \circ g\right)$ & $\left(Q_{k, h}, k \circ h\right)$ & $\left(Q_{k, k}, k \circ k\right)$ & \\
\hline$\vdots$ & & & & $\ddots$
\end{tabular}

where by $\left(Q_{g, h}, g \circ h\right)$ we mean the $|S| \times|S|$ block

\begin{tabular}{c|cccc} 
& $(r, h)$ & $(s, h)$ & $(t, h)$ & $\cdots$ \\
\hline$(r, g)$ & $(r r, g \circ h)$ & $(r s, g \circ h)$ & $(r t, g \circ h)$ & \\
$(s, g)$ & $(s r, g \circ h)$ & $(s s, g \circ h)$ & $(s t, g \circ h)$ & \\
$(t, g)$ & $(t r, g \circ h)$ & $(t s, g \circ h)$ & $(t t, g \circ h)$ & \\
$\vdots$ & & & & $\ddots$
\end{tabular}

We now attempt to turn the quasigroup amalgam into a loop by adjoining a new neutral element 1 .

Firstly, we set $1 x=x 1=x$ for every $x \in(S \times G) \cup\{1\}$. Secondly, in order to make sure that 1 appears in every row and every column precisely once, we select a bijection $c: G \rightarrow G$, for every $g \in G$ we select a loop $L_{g}$ defined on $S \cup\{1\}$ with neutral element 1 , and we replace each block $\left(Q_{g, c(g)}, g \circ c(g)\right)$ with the block $\left(L_{g}, g \circ c(g)\right)$ from which the row and column corresponding to 1 has been removed. Finally, we identity all elements of the form $(1, g)$ with 1.

The process is illustrated in Figure 1, where the underlying quasigroup $(G, \circ)$, the quasigroups $Q_{g, h}$ and the loops $L_{g}$ are chosen as follows

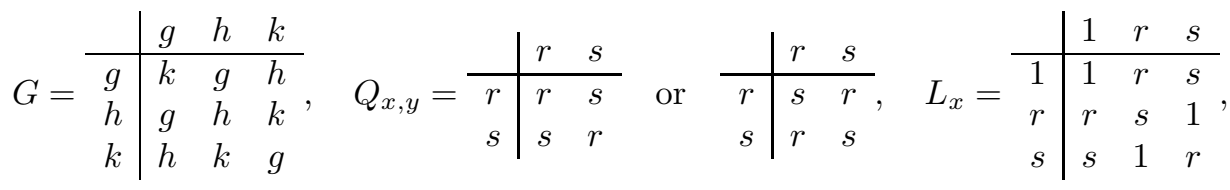

and where the bijection $c: G \rightarrow G$ is $c(g)=h, c(h)=k, c(k)=g$.

Although it might appear that the resulting quasigroup should be a loop, Figure 1 shows otherwise. Here are the conditions that make the construction work: 


\begin{tabular}{|c|cc|cc|cc|}
\hline$(r, k)$ & $(s, k)$ & $(\mathbf{r}, \mathbf{g})$ & $(\mathbf{s}, \mathbf{g})$ & $(s, h)$ & $(r, h)$ \\
$(s, k)$ & $(r, k)$ & $(\mathbf{s}, \mathbf{g})$ & $(\mathbf{r}, \mathbf{g})$ & $(r, h)$ & $(s, h)$ \\
\hline$(s, g)$ & $(r, g)$ & $(s, h)$ & $(r, h)$ & $(\mathbf{s}, \mathbf{k})$ & $(\mathbf{r}, \mathbf{k})$ \\
$(r, g)$ & $(s, g)$ & $(r, h)$ & $(s, h)$ & $(\mathbf{r}, \mathbf{k})$ & $(\mathbf{s}, \mathbf{k})$ \\
\hline$(\mathbf{r}, \mathbf{h})$ & $(\mathbf{s}, \mathbf{h})$ & $(r, k)$ & $(s, k)$ & $(s, g)$ & $(r, g)$ \\
$(\mathbf{s}, \mathbf{h})$ & $(\mathbf{r}, \mathbf{h})$ & $(s, k)$ & $(r, k)$ & $(r, g)$ & $(s, g)$ \\
\hline \multicolumn{1}{|c|}{$\downarrow$} \\
\begin{tabular}{|c|cc|cc|cc|}
\hline 1 & $(r, g)$ & $(s, g)$ & $(r, h)$ & $(s, h)$ & $(r, k)$ & $(s, k)$ \\
\hline$(r, g)$ & $(r, k)$ & $(s, k)$ & $(\mathbf{s}, \mathbf{g})$ & $\mathbf{1}$ & $(s, h)$ & $(r, h)$ \\
$(s, g)$ & $(s, k)$ & $(r, k)$ & $\mathbf{1}$ & $(\mathbf{r}, \mathbf{g})$ & $(r, h)$ & $(s, h)$ \\
\hline$(r, h)$ & $(s, g)$ & $(r, g)$ & $(s, h)$ & $(r, h)$ & $(\mathbf{s}, \mathbf{k})$ & $\mathbf{1}$ \\
$(s, h)$ & $(r, g)$ & $(s, g)$ & $(r, h)$ & $(s, h)$ & $\mathbf{1}$ & $(\mathbf{r}, \mathbf{k})$ \\
\hline$(r, k)$ & $(\mathbf{s}, \mathbf{h})$ & $\mathbf{1}$ & $(r, k)$ & $(s, k)$ & $(s, g)$ & $(r, g)$ \\
$(s, k)$ & $\mathbf{1}$ & $(\mathbf{r}, \mathbf{h})$ & $(s, k)$ & $(r, k)$ & $(r, g)$ & $(s, g)$ \\
\hline
\end{tabular}
\end{tabular}

FiguRE 1. Attempting to create a loop out of a quasigroup amalgam by adjoining 1

Proposition 3.1. Let $G, L_{g}, Q_{g, h}$ and $c: G \rightarrow G$ be as above. Then the resulting magma is a loop if and only if $c$ is the identity on $G$ and $G$ is an idempotent quasigroup.

Proof. We show the necessity of the conditions and leave the sufficiency to the reader. Assume that the resulting magma is a loop. Let $g \in G$ and $s \in S$. Let $k \in G$ be such that $g \circ k=g$. If $c(g) \neq k$, then the block $\left(Q_{g, k}, g \circ k\right)$ has not been replaced, and thus it contains $(s, g \circ k)=(s, g)$ in the row labeled by $(s, g)$, a contradiction with $(s, g) 1=(s, g)$. Hence $g \circ c(g)=g$. Suppose that $g \neq c(g)$. Then there is $h \neq g$ such that $h \circ c(g)=c(g)$. Since the block $\left(Q_{h, c(g)}, h \circ c(g)\right)$ has not been replaced, it contains $(s, h \circ c(g))=(s, c(g))$ in the column labeled by $(s, c(g))$, a contradiction with $1(s, c(g))=(s, c(g))$. Thus $g=c(g)$.

We therefore define the loop amalgam as follows: Let $(G, \circ)$ be an idempotent quasigroup, $S$ a nonempty set, and 1 an element not contained in $S$. Let $\mathcal{Q}=$ $\left\{Q_{g, h} \mid g, h \in G, g \neq h\right\}$ be a family of quasigroups with underlying set $S$. Let $\mathcal{L}=\left\{L_{g} \mid g \in G\right\}$ be a family of loops with underlying set $S \cup\{1\}$ and with neutral element 1 . Then the (loop) amalgam $\mathcal{A}=\mathcal{A}(G, \mathcal{L}, \mathcal{Q})$ is a magma defined on the set $X=(S \times G) \cup\{1\}$ by

$$
\begin{gathered}
1 x=x 1=x \text { for every } x \in X, \\
(s, g)(t, h)=(s t, g \circ h) \text { for every } s, t \in S, g, h \in G,
\end{gathered}
$$

where we identify the elements $(1, g)$ with 1 , and where the multiplication st takes place in $L_{g}$ when $g=h$, and in $Q_{g, h}$ when $g \neq h$.

The following result is then easily observed:

Lemma 3.2. The amalgam $\mathcal{A}(G, \mathcal{L}, \mathcal{Q})$ is commutative if and only if $(G, \circ)$ is commutative, each $L_{g}$ is commutative, and $Q_{g, h}$ is the opposite quasigroup of $Q_{h, g}$ for every $g \neq h \in G$. In addition, $|\mathcal{A}|=|G||S|+1=|G|\left(\left|L_{g}\right|-1\right)+1$. 
Note that $\mathcal{Q}=\emptyset$ if $G=\{x\}$ is a singleton quasigroup. Any loop $L$ can then be obtained as an amalgam by letting $G=\{x\}$ and $L_{x}=L$, a situation that we call trivial.

A restricted version of the amalgam construction appears in [5. Namely, Foguel lets $(G, \circ)$ be an idempotent quasigroup, sets $L_{g}=L$ for every $g \in G$, and $Q_{g, h}=Q$ for every $g \neq h \in G$. We denote his construction by $\mathcal{A}(G, L, Q)$.

Proposition 3.3. Let $(G, \circ)$ be an idempotent quasigroup, $(L, \bullet)$ a loop, and $(Q, *)$ a quasigroup defined on $L \backslash\{1\}$. Then $\mathcal{A}=\mathcal{A}(G, L, Q)$ is a Jordan loop if and only if

(i) L is a Jordan loop, and

(ii) $G$ and $Q$ are commutative, and

(iii) for every $s, t \in Q$ either $s \bullet s=1$ or $(s \bullet s) *(t * s)=((s \bullet s) * t) * s$.

Proof. We know from Lemma 3.2 that $\mathcal{A}$ is commutative if and only if $G, L$ and $Q$ are commutative.

Let $x=(s, g) \neq 1 \neq(t, h)=y \in \mathcal{A}$. If $g=h$ then (J) holds for $x, y$ if and only if $(s \bullet s) \bullet(t \bullet s)=((s \bullet s) \bullet t) \bullet s$.

Assume that $g \neq h$. Then

$$
\begin{aligned}
& x^{2}(y x)=(s \bullet s, g)(t * s, h \circ g), \\
& \left(x^{2} y\right) x=(s \bullet s, g)(t, h) \cdot(s, g) .
\end{aligned}
$$

If $s \bullet s=1$, the two expressions are equal. If $s \bullet s \neq 1$, then

$$
\begin{aligned}
& x^{2}(y x)=((s \bullet s) *(t * s), g \circ(h \circ g)), \\
& \left(x^{2} y\right) x=((s \bullet s) * t, g \circ h)(s, g)=(((s \bullet s) * t) * s,(g \circ h) \circ g) .
\end{aligned}
$$

Thus, when $G$ is commutative, the two expressions are equal if and only if $(s \bullet s) *$ $(t * s)=((s \bullet s) * t) * s$.

Proposition 3.4. Let $n>5$ be an odd integer not of the form $2^{m}+1$. Then there exists a Jordan loop of order $n$ which is not left alternative, and hence, is not associative.

Proof. Since $n-1 \neq 2^{m}$, we have $n-1=2^{\ell} k$ where $k \geq 3$ is odd and $\ell \geq 0$. Let $(G, \circ)$ be a commutative idempotent quasigroup of order $k$, which exists by Lemma 2.6. Let $(L, \bullet)$ be a commutative group of order $2^{\ell}+1$, and $(Q, *)$ a commutative group of order $2^{\ell}$ defined on $L \backslash\{1\}$.

Set $\mathcal{A}=\mathcal{A}(G, L, Q)$. Then $|\mathcal{A}|=n$ by Lemma 3.2 , and $\mathcal{A}$ is a Jordan loop by Proposition 3.3

Choose distinct elements $g, h \in G$ and an element $s \in L$ with $s \neq 1$. Then $g=g \circ g \neq g \circ h$, and since $L$ is a group of odd order, $s \bullet s \neq 1$. Thus

$$
\begin{aligned}
& (s, g)(s, g) \cdot(s, h)=(s \bullet s, g)(s, h)=((s \bullet s) * s, g \circ h), \\
& (s, g) \cdot(s, g)(s, h)=(s, g)(s * s, g \circ h)=(s *(s * s), g \circ(g \circ h)) .
\end{aligned}
$$

As $g \neq g \circ h$, we have $g \circ h=(g \circ h) \circ(g \circ h) \neq g \circ(g \circ h)$, and it follows that $\mathcal{A}$ is not left alternative. 


\section{ORDER $2^{m}+1$}

Assume momentarily that $\mathcal{A}(G, \mathcal{L}, \mathcal{Q})$ is a commutative loop of order $2^{m}+1$ and $|G|>1$. Then, by Lemma 3.2, $G$ is a commutative idempotent quasigroup of even order, which contradicts Lemma 2.6. It is therefore impossible to construct a commutative loop of order $2^{m}+1$ as a nontrivial amalgam.

When we arrived at this impasse, we seriously entertained the possibility that there are infinitely many orders $n$ for which no nonassociative Jordan loops exist. We soon noticed that this alternative has interesting number-theoretical consequences. We were unable to resist the temptation to present them here, despite the fact that Theorem 1.1 renders them irrelevant.

Observation 4.1. Assume that there are infinitely many orders $n$ for which no nonassociative Jordan loop exists. Then there are infinitely many Fermat primes, i.e., primes of the form $2^{m}+1$.

Proof. The results of $₫ 2$ and Proposition 3.4 imply that under our assumption there are infinitely many orders $n=2^{m}+1$ for which no nonassociative Jordan loop exists. Let $p_{1}^{a_{1}} \cdots p_{k}^{a_{k}}$ be a prime factorization of such an $n=2^{m}+1$.

If some $p_{i}$ is not Fermat, then $p_{i}>5$, there is a nonassociative Jordan loop $Q$ of order $p_{i}$ by Proposition 3.4, and hence a nonassociative Jordan loop of order $n$ is obtained as a direct product of $Q$ with a commutative group of order $n / p_{i}$, a contradiction. Thus every $p_{i}$ is a Fermat prime.

If there is an $i$ such that $p_{i}>3$ and $a_{i}>1$ then $p_{i}^{2}=\left(2^{r}+1\right)^{2}$ is not of the form $2^{s}+1$, as can be routinely observed, so there is a nonassociative Jordan loop $Q$ of order $p_{i}^{2}$ by Proposition 3.4 again leading to a contradiction. Hence for every $i$ we have $p_{i} \leq 3$ or $a_{i}=1$.

If there is an $i$ such that $p_{i}=3$ and $a_{i}>2$ then $p_{i}^{3}=27$ divides $n$ but is not of the form $2^{s}+1$, a contradiction once again.

Altogether, we see that every $p_{i}$ is a Fermat prime, if $p_{i}>3$ then $a_{i}=1$, and if $p_{i}=3$ then $a_{i} \leq 2$. Hence there must be infinitely many Fermat primes.

Since it is generally (but not universally) believed that (i) there are finitely many Fermat primes, and (ii) it is hard to establish (i), we concluded that the premise of Observation 4.1 is likely false. And, indeed, a modification of the amalgam construction allowed us to settle the case $2^{m}+1$, too.

In 3 , we showed how to turn the quasigroup amalgam $\mathcal{A}=\mathcal{A}(G, \mathcal{Q})$ into a loop by adjoining a neutral element 1 and replacing the diagonal blocks $\left(Q_{g, g}, g\right)$ by $\left(L_{g}, g\right)$. The underlying principle that makes this work is the fact that $\mathcal{A}$ is a quasigroup that is a disjoint union of (some of its) subquasigroups, namely $\mathcal{A}=$ $\bigcup_{g \in G}\left(Q_{g, g}, g\right)$.

This leads us to a more general construction: Let $Q=\bigcup_{i \in I} Q_{i}$ be a quasigroup, where $Q_{i} \leq Q$ and $Q_{i} \cap Q_{j}=\emptyset$ for every $i \neq j \in I$. Let 1 be an element not contained in $Q$. For every $i \in I$, let $L_{i}$ be a loop defined on $Q_{i} \cup\{1\}$. Upon replacing the blocks $Q_{i} \times Q_{i}$ with $\left(L_{i} \backslash\{1\}\right) \times\left(L_{i} \backslash\{1\}\right)$ in the multiplication table of $Q$ and upon adjoining 1 as the neutral element, we obtain a loop.

Instead of introducing more notation, we say, somewhat informally, that the resulting loop is obtained from $Q$ by replacing the subquasigroups $Q_{i}$ with subloops $L_{i}$.

Let us a call a quasigroup Jordan if it is commutative and satisfies (J). 
Lemma 4.2. Let $G$ be a commutative group, $G_{i} \leq G$ for $i \in I, G_{i} \cap G_{j}=\{1\}$ for $i \neq j \in I$, and $G=\bigcup_{i \in I} G_{i}$. Let $Q=G \backslash\{1\}, Q_{i}=G_{i} \backslash\{1\}$, and assume that $\left(Q_{i}, *\right)$ is a Jordan quasigroup for every $i \in I$. Then $(Q, *)$ with multiplication defined by

$$
x * y= \begin{cases}x * y, & \text { if } x, y \in Q_{i}, \\ x y, & \text { if } x \in Q_{i}, y \in Q_{j}, i \neq j\end{cases}
$$

is a Jordan quasigroup, $Q_{i} \leq Q, Q_{i} \cap Q_{j}=\emptyset$ for $i \neq j$, and $Q=\bigcup_{i \in I} Q_{i}$.

Proof. We leave it to the reader to verify that $(Q, *)$ is a commutative quasigroup. Let us check (J). Let $x, y \in Q$. If there is $i \in I$ such that $x \in Q_{i}$ and $y \in Q_{i}$, then the verification of $(x * x) *(y * x)=((x * x) * y) * x$ takes place entirely within the Jordan quasigroup $\left(Q_{i}, *\right)$. Assume that $x \in Q_{i}, y \in Q_{j}$, and $i \neq j$. Since $Q_{i} Q_{j} \cap Q_{i}=\emptyset$ and $Q_{i} * Q_{i} \subseteq Q_{i}$, we have

$$
\begin{aligned}
& (x * x) *(y * x)=(x * x) *(y x)=(x * x)(y x), \\
& ((x * x) * y) * x=((x * x) y) * x=((x * x) y) x .
\end{aligned}
$$

We are through because $G$ is associative.

Proposition 4.3. For every $m>3$ there is a nonassociative Jordan loop of order $2^{m}+1$.

Proof. Let $G=\left\langle\alpha, \beta ; \alpha^{3}=\beta^{3}=1, \alpha \beta=\beta \alpha\right\rangle$ be the direct product of the cyclic group of order 3 with itself. Let $G_{1}=\langle\alpha\rangle, G_{2}=\langle\beta\rangle, G_{3}=\langle\alpha \beta\rangle, G_{4}=\left\langle\alpha \beta^{2}\right\rangle$. Then $G_{i} \cap G_{j}=\{1\}$ for $i \neq j$, and $G=\bigcup_{1 \leq i \leq 4} G_{i}$.

For $Q_{i}=G_{i} \backslash\{1\}$, let $\left(Q_{i}, *\right)$ be the cyclic group of order 2 . Let $(Q, *)$ be the Jordan quasigroup (of order 8) obtained by Lemma 4.2.

Let $C$ be the cyclic group of order $2^{m-3}$, and set $(\bar{Q}, *)=C \times Q$. Then $\bar{Q}$ is a Jordan quasigroup of order $2^{m}$ that is a disjoint union of its subquasigroups $\overline{Q_{i}}=C \times Q_{i}$, each of order $2^{m-2}$.

Let $L$ be a commutative group of order $2^{m-2}+1$, and let $J$ be the loop obtained from $\bar{Q}$ by replacing the subquasigroups $\overline{Q_{i}}$ with copies of $L$. Clearly, $|J|=2^{m}+1$, $J$ is commutative (because $Q$ is), and we claim that $J$ is a nonassociative Jordan loop.

We first check that $J$ satisfies the Jordan identity $x^{2}(y x)=\left(x^{2} y\right) x$. If $x=1$ or $y=1$, there is nothing to prove. If $x, y$ belong to the same subquasigroup $\overline{Q_{i}}$ of $\bar{Q}$, then the verification of $x^{2}(y x)=\left(x^{2} y\right) x$ takes place entirely within a copy of the group $L$. Assume that $x \in \overline{Q_{i}}, y \in \overline{Q_{j}}, i \neq j$. Since $L$ contains no elements of order 2 , we have $x^{2} \in \overline{Q_{i}}$. Then $x^{2}(y x)=x^{2}(y * x)=x^{2} *(y * x)$, and $\left(x^{2} y\right) x=\left(x^{2} * y\right) x=\left(x^{2} * y\right) * x$. As $(\bar{Q}, *)$ is Jordan, (J) holds for $J$, too.

Since $L$ is a subloop of $J,|L|=2^{m-2}+1,|J|=2^{m}+1$, and $2^{m-2}+1$ does not divide $2^{m}+1$ for $m>3$, it follows that $J$ cannot be a group, by the Lagrange theorem.

The restriction that $m>3$, which is not used until the last paragraph of the proof of Proposition 4.3 cannot be removed. Indeed, it turns out that no nonassociative Jordan loop of order 5 or 9 exists. In the next section we give an easy proof of this assertion for order 5 . We were not able to find a short, human argument for order 9. However, a finite model builder, such as Mace4 [9], shows that no such loop exists. Theorem 1.1 will then be established. 


\section{Powers in JordAN LOOPS}

Let $Q$ be a loop and $c \in Q$. We use the right-associated convention for powers: $c^{0}=1, c^{n}=c c^{n-1}$ for $n \geq 1$. For $n>0$, we say that $c^{n}$ is well-defined if the value of $c \cdots c$ is independent of how the $n$ factors are parenthesized. This has an obvious formal characterization, which could also be taken as an inductive definition.

Lemma 5.1. Let $Q$ be a loop and $c \in Q$. For $n>0, c^{n}$ is well-defined if and only if, for each $0<k<n, c^{k}$ is well-defined and $c^{k} c^{n-k}=c^{n}$.

Lemma 5.2. Let $Q$ be a Jordan loop and $c \in Q$. Then $c^{n}$ is well-defined whenever $1 \leq n \leq 5$.

Proof. Commutativity alone shows that $c^{3}$ is well-defined. By (J), $c^{2} \cdot c c=c^{2} c \cdot c$, and hence $c^{4}$ is well-defined. Finally, $c^{2} c^{3}=c^{2} \cdot c^{2} c=c^{2} c^{2} \cdot c$ by (J), and so $c^{5}$ is well-defined.

Lemma 5.3. Let $Q$ be a loop of order $n$ and let $c \in Q$. If $c^{m}$ is well-defined for every $1 \leq m \leq n-1$ then $\langle c\rangle$ is a cyclic group of order $k$, and $k=n$ whenever $k>\lfloor n / 2\rfloor$.

Proof. The $(n+1)$-tuple $\left(1, c, \ldots, c^{n-1}, c \cdot c^{n-1}\right)$ contains a repetition. Upon canceling $c$ on the left as many times as needed, we conclude that there is $1 \leq k \leq n$ such that $1, c, \ldots, c^{k-1}$ are distinct and $c c^{k-1}=1$. (We write $c c^{k-1}$ rather than $c^{k}$ because $k=n$ could occur and we do not know yet if $c^{n}$ is well-defined.) Let $\left\{a_{1}, \ldots, a_{n-k}\right\}=Q \backslash\left\{1, c, \ldots, c^{k-1}\right\}$.

Assume that $k<n$. We have $c^{i} c^{j}=c^{(i+j)} \bmod k$ for every $0 \leq i \leq k-1$, $0 \leq j \leq n-k$, as $c^{k}=1$. If $2 k \leq n+1$, it immediately follows that $\langle c\rangle$ is a cyclic group of order $k$. If $2 k \geq n$, the nonempty $(n-k) \times(n-k+1)$ block with rows labeled by $a_{1}, \ldots, a_{n-k}$ and columns labeled by $1, c, \ldots, c^{n-k}$ must contain only the elements $a_{1}, \ldots, a_{n-k}$, a contradiction.

Now assume that $k=n$, the elements of $Q$ are listed as $1, c, \ldots, c^{n-1}$, and $c c^{n-1}=1$. We prove by induction on $i$ that $c^{i} c^{j}=c^{i+j} \bmod n$ for every $0 \leq i$, $j<n$. There is nothing to show when $i=0$, and the statement also holds for $i=1$ thanks to $c c^{n-1}=1$. Assume that the statement is true for $i-1 \geq 1$, and consider the row labeled by $c^{i}$, as visualized below:

\begin{tabular}{c|cccccccc} 
& 1 & $c$ & $\ldots$ & $c^{n-i-1}$ & $c^{n-i}$ & $\ldots$ & $c^{n-2}$ & $c^{n-1}$ \\
\hline$c^{i}$ & $c^{i}$ & $c^{i+1}$ & $\ldots$ & $c^{n-1}$ & $?$ & $?$ & $?$ & $?$
\end{tabular}

We certainly have $c^{i} c^{n-1} \in\left\{1, c, \ldots, c^{i-1}\right\}$, and since each of $1, c, \ldots, c^{i-2}$ already occurs in the last column, we in fact have $c^{i} c^{n-1}=c^{i-1}$. Proceeding from right to left, we complete the row as claimed. In particular, $c^{i} c^{n-i}=1$ since no other power of $c$ is available at that point.

Corollary 5.4. Every Jordan loop of order 5 is a cyclic group.

Proof. Let $Q$ be such a Jordan loop, and fix $c \in Q$ with $c \neq 1$. By Lemma 5.2, $c^{k}$ is well-defined for $1 \leq k \leq 5$. By Lemma [5.3, $\langle c\rangle$ is a cyclic group of order 2 or 5 . But by Corollary 2.4, $c$ cannot have order 2. Thus $Q=\langle c\rangle$.

It is not difficult to use Lemma 5.3 to classify Jordan loops of orders 6 and 7. It turns out that there is only one nonassociative Jordan loop of order 6 , and it is the commutative loop of exponent 2 constructed in $\$ 2$. There are only two 
nonassociative Jordan loops of order 7, and each element of those loops has order 3 . One of them is given by the amalgam construction of $\$ 3$, and the other is given by the construction of 86 . Details will appear elsewhere [11].

The case of Jordan loops of order 9 is trickier. It turns out that the only two such loops are the two groups. A complete, and rather long, human proof of this will appear in [11. In the meantime, it is easy to use a finite model builder such as Mace4 9] to search exhaustively for Jordan loops of small orders, and such a search quickly shows that there are no nonassociative models of order 9 . With this caveat in place, this completes the proof of Theorem 1.1

It is an interesting problem to determine which powers of elements in Jordan loops are well-defined. We give a complete answer for those powers greater than 5 which are neither odd primes nor powers of 2 .

Theorem 5.5. Let $m, n$ be integers with $m \geq 2, n \geq 3$, and $n$ odd. Then there exists a 1-generated Jordan loop $Q$ with a generator $c$ such that $c^{k}$ is well-defined for $0 \leq k<m n$, but $c^{p n} c^{(m-p) n} \neq c^{m n}$ for $0<p<m$.

Proof. Let $s \geq m+2$ be the smallest positive integer relatively prime to $n$.

For an integer $\ell$, we denote by $\bar{\ell}$ its image in $\mathbb{Z}_{n}=\mathbb{Z} / n \mathbb{Z}$ and by $[\ell]$ the image in $\mathbb{Z}_{s}$. Then $[n]$ is a generator of $\mathbb{Z}_{s}=\{[0],[n], \ldots,(s-1)[n]\}$.

We define a permutation $\phi$ on $\mathbb{Z}_{s}$ by

$$
\phi(i[n])= \begin{cases}i[n], & \text { if } 0 \leq i \leq m-1 \\ (s+m-i-1)[n], & \text { if } m \leq i \leq s-1\end{cases}
$$

Using $\phi$, we define a new operation $\circ$ on $\mathbb{Z}_{s}$ by

$$
[k] \circ[\ell]=\phi^{-1}(\phi([k])+\phi([\ell]))
$$

for all $k, l \in \mathbb{Z}$. (The assumption that $s \geq m+2$ is used here to guarantee that $\phi(m[n]) \neq m[n]$.$) Then \left(\mathbb{Z}_{s}, \circ\right)$ is an isomorphic copy of $\left(\mathbb{Z}_{s},+\right)$.

Now let $Q=\mathbb{Z}_{n} \times \mathbb{Z}_{s}$, and define a binary operation $\cdot$ on $Q$ as follows. For $a, b, u, v \in \mathbb{Z}$, set

$$
(\bar{a},[u]) \cdot(\bar{b},[v])= \begin{cases}(\overline{a+b},[u+v]) & \text { if } a \not \equiv 0 \text { or } b \not \equiv 0 \bmod n \\ (\overline{0},[u] \circ[v]) & \text { if } a \equiv b \equiv 0 \bmod n\end{cases}
$$

It is clear that $(Q, \cdot)$ is a commutative loop with neutral element $(0,0)$. We claim that $(Q, \cdot)$ is, in fact, a Jordan loop, that is, it satisfies

$$
(\bar{a},[u])^{2} \cdot(\bar{b},[v])(\bar{a},[u])=(\bar{a},[u])^{2}(\bar{b},[v]) \cdot(\bar{a},[u])
$$

for all $a, b, u, v \in \mathbb{Z}$.

Firstly, assume $a \not \equiv 0 \bmod n$. Then $(\bar{a},[u])^{2}=(\overline{2 a},[2 u])$. Since $n$ is odd, $2 a \not \equiv 0$ $\bmod n$, and so

$$
\begin{aligned}
(\bar{a},[u])^{2}(\bar{b},[v]) \cdot(\bar{a},[u]) & =(\overline{2 a+b},[2 u+v])(\bar{a},[u])=(\overline{3 a+b},[3 u+v]) \\
& =(2 a,[2 u]) \cdot(\bar{b},[v])(\bar{a},[u])=(\bar{a},[u])^{2} \cdot(\bar{b},[v])(\bar{a},[u]) .
\end{aligned}
$$

Next, assume $a \equiv 0 \bmod n$. Then $(\overline{0},[u])^{2}=(\overline{0},[u] \circ[u])$. If $b \not \equiv 0 \bmod n$, then

$$
\begin{aligned}
(\overline{0},[u])^{2} \cdot(\bar{b},[v])(\overline{0},[u]) & =(\overline{0},[u] \circ[u]) \cdot(\bar{b},[v+u])=(\bar{b},[([u] \circ[u])+v+u]) \\
& =(\bar{b},[([u] \circ[u])+v])(\overline{0},[u])=(\overline{0},[u])^{2}(\bar{b},[v]) \cdot(\overline{0},[u]) .
\end{aligned}
$$


The identity also holds if $b \equiv 0 \bmod n$, for then all calculations in the second component occur in the cyclic group $\left(\mathbb{Z}_{s}, \circ\right)$. This completes the verification that $(Q, \cdot)$ is a Jordan loop.

Set $c=(\overline{1},[1]) \in Q$. Then for all $k>0, c^{k}=(\bar{k},[k])$. (Recall that $c^{k}$ is the right associated product $c(c \cdots c)$.) We claim that $c$ generates $Q$. Indeed, for $a, b \in \mathbb{Z}$, choose $p, q, r, t \in \mathbb{Z}$ such that $p n+q s=a$ and $r n+t s=b$. Then $c^{q s} c^{r n}=(\overline{q s},[0])(\overline{0},[r n])=(\overline{q s},[r n])=(\bar{a},[b])$.

Next we wish to show that $c^{k}$ is well-defined for $0 \leq k<m n$. Thus we must show that $c^{i} c^{j}=c^{i+j}$ for $0 \leq i+j \leq m n-1$.

If at least one of $i, j$ is not divisible by $n$, then $c^{i} c^{j}=(\bar{i},[i])(\bar{j},[j])=(\overline{i+j},[i+$ $j])=c^{i+j}$. For the other case, assume that $i=p n$ and $j=q n$, where $0 \leq$ $p, q, p+q \leq m-1$. Then

$$
[i] \circ[j]=\phi^{-1}(\phi(p[n])+\phi(q[n]))=\phi^{-1}((p+q)[n])=(p+q)[n]=[i+j] .
$$

Therefore $c^{i} c^{j}=(\overline{0},[i])(\overline{0},[j])=(\overline{0},[i] \circ[j])=(\overline{0},[i]+[j])=c^{i+j}$ in this case as well.

Finally, suppose $0<p<m$. Then $m-p \leq m-1$, and so $\phi^{-1}(\phi(p[n])+\phi((m-$ $p)[n]))=\phi^{-1}(p[n]+(m-p)[n])=\phi^{-1}(m[n])=(s-1)[n]$. Therefore,

$$
\begin{aligned}
c^{p n} c^{(m-p) n} & =(\overline{0}, p[n])(\overline{0},(m-p)[n])=(\overline{0}, p[n] \circ(m-p)[n]) \\
& =(\overline{0},(s-1)[n]) \neq(\overline{0}, m[n])=c^{m n},
\end{aligned}
$$

as claimed.

To illustrate the construction of Theorem 5.5. consider the particular case $n=$ $3, m=2$. In this case, $s=4$, and so the order of the loop will be 12 . The permutation $\phi$ is given by $\phi([0])=[0], \phi([3])=[3], \phi([2])=\phi(2[3])=3[3]=[1]$, $\phi([1])=\phi(3[3])=2[3]=[2]$. The operation $\circ$ on $\mathbb{Z}_{4}$ is given by

\begin{tabular}{c|cccc}
$\circ$ & {$[0]$} & {$[1]$} & {$[2]$} & {$[3]$} \\
\hline$[0]$ & {$[0]$} & {$[1]$} & {$[2]$} & {$[3]$} \\
{$[1]$} & {$[1]$} & {$[0]$} & {$[3]$} & {$[2]$} \\
{$[2]$} & {$[2]$} & {$[3]$} & {$[1]$} & {$[0]$} \\
{$[3]$} & {$[3]$} & {$[2]$} & {$[0]$} & {$[1]$}
\end{tabular}

We represent the loop element $(\bar{a},[u])$ by the nonnegative integer $a+3 u$. Then the loop multiplication table is as follows.

\begin{tabular}{ccc|ccc|ccc|ccc}
0 & 1 & 2 & 3 & 4 & 5 & 6 & 7 & 8 & 9 & 10 & 11 \\
1 & 2 & 0 & 4 & 5 & 3 & 7 & 8 & 6 & 10 & 11 & 9 \\
2 & 0 & 1 & 5 & 3 & 4 & 8 & 6 & 7 & 11 & 9 & 10 \\
\hline 3 & 4 & 5 & 0 & 7 & 8 & 9 & 10 & 11 & 6 & 1 & 2 \\
4 & 5 & 3 & 7 & 8 & 6 & 10 & 11 & 9 & 1 & 2 & 0 \\
5 & 3 & 4 & 8 & 6 & 7 & 11 & 9 & 10 & 2 & 0 & 1 \\
\hline 6 & 7 & 8 & 9 & 10 & 11 & 3 & 1 & 2 & 0 & 4 & 5 \\
7 & 8 & 6 & 10 & 11 & 9 & 1 & 2 & 3 & 4 & 5 & 6 \\
8 & 6 & 7 & 11 & 9 & 10 & 2 & 3 & 4 & 5 & 6 & 7 \\
\hline 9 & 10 & 11 & 6 & 1 & 2 & 0 & 4 & 5 & 3 & 7 & 8 \\
10 & 11 & 9 & 1 & 2 & 0 & 4 & 5 & 6 & 7 & 8 & 6 \\
11 & 9 & 10 & 2 & 0 & 1 & 5 & 6 & 7 & 8 & 6 & 7
\end{tabular}

The generator $c$ of Theorem 5.5 is 4 , and we see that $4(4 \cdot 4) \cdot 4(4 \cdot 4)=3 \neq 6=$ $4 \cdot 4(4 \cdot 4(4 \cdot 4))$. 
Whether $x^{m}$ where $m>6$ is an odd prime or a power of 2 is well-defined is at present unclear. For instance, in a Jordan loop, if $c$ is an element such that $c^{6}$ is well-defined, then both $c^{7}$ and $c^{8}$ are well-defined. If a Jordan loop $Q$ satisfies $x^{m}$ is well-defined for all $x \in Q$ for $0 \leq m \leq 10$, then $x^{11}$ is also well-defined for all $x \in Q$. We do not know of an example of a Jordan loop in which there exists an element $c$ with $c^{m}$ well-defined for $0 \leq m \leq 10$, but such that $c^{11}$ is not well-defined.

Problem 5.6. Let $Q$ be a Jordan loop, and let $m>6$ be an integer which is either an odd prime or a power of 2 .

(i) For a fixed $c \in Q$, if $c^{k}$ is well-defined for $0 \leq k<m$, must $c^{m}$ be welldefined?

(ii) If $x^{k}$ is well-defined for all $x \in Q$ and for $0 \leq k<m$, must $x^{m}$ be welldefined?

Problem 5.7. For which $n$ does there exist a 1-generated Jordan loop of order $n$ which is not power associative? In particular, is there a 1-generated Jordan loop of prime order or of order a power of 2 which is not power associative?

\section{An INFINIte FAMily of NONASSOCIATIVE SIMPlE JORDAN LOOPS}

In this section we construct an infinite family of nonassociative simple Jordan loops. Although the construction could be viewed as another generalized amalgam, we opt for a more direct description.

Let $A=(A, \cdot)$ be a commutative loop of order $2^{n}-1 \geq 1$, and let

$$
B=B_{n}=\left\{\left[x_{1}, \ldots, x_{n}\right]=\left[x_{i}\right] ; x_{i} \in\{0,1\}\right\}
$$

be the binary hypercube of size $2^{n}$. Label elements of $A$ by $\left(x_{i}\right)$, where $\left[x_{i}\right]$ is any element of $B$ different from $[1, \ldots, 1]$, and let $(0, \ldots, 0)$ be the neutral element of $A$. (As a mnemonic device, loop elements are enclosed in round parentheses, hypercube elements in brackets.)

Define an operation $\circ$ on the disjoint union $A \cup B$ by

$$
\begin{aligned}
& \left(x_{i}\right) \circ\left(y_{i}\right)=\left(x_{i}\right) \cdot\left(y_{i}\right), \\
& \left(x_{i}\right) \circ\left[y_{i}\right]=\left[x_{i} \oplus y_{i}\right], \\
& {\left[x_{i}\right] \circ\left(y_{i}\right)=\left[x_{i} \oplus y_{i}\right] \text {, }} \\
& {\left[x_{i}\right] \circ\left[y_{i}\right]= \begin{cases}{\left[x_{i}^{\prime}\right],} & \text { if }\left[x_{i}\right]=\left[y_{i}\right], \\
\left(\left(x_{i} \oplus y_{i}\right)^{\prime}\right), & \text { otherwise }\end{cases} }
\end{aligned}
$$

where $\oplus$ is the usual binary addition, and ' is the binary complement.

Note that $\left[\left(x_{i} \oplus y_{i}\right)^{\prime}\right]$ is different from $[1, \ldots, 1]$ when $\left[x_{i}\right] \neq\left[y_{i}\right]$, and hence $\circ$ is indeed an operation on $A \cup B$, clearly commutative. We will denote the resulting groupoid by $\mathcal{J}(A)$.

Before we analyze the construction, it is once again worth visualizing it in terms of a multiplication table.

Here is $\mathcal{J}(1)$ for the trivial one-element loop 1 :

\begin{tabular}{c||c|cc}
$\circ$ & $(0)$ & {$[0]$} & {$[1]$} \\
\hline \hline$(0)$ & $(0)$ & {$[0]$} & {$[1]$} \\
\hline$[0]$ & {$[0]$} & {$[1]$} & $(0)$ \\
{$[1]$} & {$[1]$} & $(0)$ & {$[0]$}
\end{tabular}


Of course, this is just the cyclic group of order 3 , hence again a commutative loop. Upon relabeling the elements of $\mathcal{J}(1)$ as $(0,0),(0,1),(1,0)$, we obtain $\mathcal{J}(\mathcal{J}(1))$ :

\begin{tabular}{c||ccc|cccc}
$\circ$ & $(0,0)$ & $(0,1)$ & $(1,0)$ & {$[0,0]$} & {$[0,1]$} & {$[1,0]$} & {$[1,1]$} \\
\hline \hline$(0,0)$ & $(0,0)$ & $(0,1)$ & $(1,0)$ & {$[0,0]$} & {$[0,1]$} & {$[1,0]$} & {$[1,1]$} \\
$(0,1)$ & $(0,1)$ & $(1,0)$ & $(0,0)$ & {$[0,1]$} & {$[0,0]$} & {$[1,1]$} & {$[1,0]$} \\
$(1,0)$ & $(1,0)$ & $(0,0)$ & $(0,1)$ & {$[1,0]$} & {$[1,1]$} & {$[0,0]$} & {$[0,1]$} \\
\hline$[0,0]$ & {$[0,0]$} & {$[0,1]$} & {$[1,0]$} & {$[1,1]$} & $(1,0)$ & $(0,1)$ & $(0,0)$ \\
{$[0,1]$} & {$[0,1]$} & {$[0,0]$} & {$[1,1]$} & $(1,0)$ & {$[1,0]$} & $(0,0)$ & $(0,1)$ \\
{$[1,0]$} & {$[1,0]$} & {$[1,1]$} & {$[0,0]$} & $(0,1)$ & $(0,0)$ & {$[0,1]$} & $(1,0)$ \\
{$[1,1]$} & {$[1,1]$} & {$[1,0]$} & {$[0,1]$} & $(0,0)$ & $(0,1)$ & $(1,0)$ & {$[0,0]$}
\end{tabular}

We can therefore think of the multiplication table for $\mathcal{J}(A)$ as consisting of four quadrants:

- the top left $2^{n}-1$ times $2^{n}-1$ quadrant is the multiplication table of $A$,

- the top right $2^{n}-1$ times $2^{n}$ quadrant is the canonical multiplication table of the elementary abelian group of order $2^{n}$ with the last row removed,

- the bottom left $2^{n}$ times $2^{n}-1$ quadrant is the canonical multiplication table of the elementary abelian group of order $2^{n}$ with the last column removed,

- the bottom right $2^{n}$ times $2^{n}$ quadrant is the canonical multiplication table of the elementary abelian group of order $2^{n}$, except that the elements are listed in reversed order (this is because of the complement in the definition), and that the elements of $B_{n}$ are superimposed on the main diagonal, again in reversed order.

A short reflection shows that the multiplication table of $\mathcal{J}(A)$ is a Latin square. We have obtained:

Proposition 6.1. Let $A$ be a commutative loop of order $2^{n}-1$, and let $\mathcal{J}(A)$ be defined as above. Then $\mathcal{J}(A)$ is a commutative loop of order $2^{n+1}-1$ with subloop $A$.

Proposition 6.2. Let $A$ be a Jordan loop. Then $\mathcal{J}(A)$ is a Jordan loop.

Proof. We must verify $(x \circ x) \circ(y \circ x)=((x \circ x) \circ y) \circ x$ for every $x, y \in \mathcal{J}(A)=A \cup B$. When $x \in A, y \in A$, there is nothing to show since $A$ is Jordan and $\circ$ coincides with the multiplication in $A$ on $A \times A$. For the rest of the proof, let $x=\left(x_{i}\right)$ or $x=\left[x_{i}\right]$, and $y=\left(y_{i}\right)$ or $y=\left[y_{i}\right]$.

Assume that $x \in A$ and $y \in B$. Let $\left(z_{i}\right)=x \circ x$. Then $(x \circ x) \circ(y \circ x)=$ $\left(\left(x_{i}\right) \circ\left(x_{i}\right)\right) \circ\left(\left[y_{i}\right] \circ\left(x_{i}\right)\right)=\left(z_{i}\right) \circ\left[y_{i} \oplus x_{i}\right]=\left[z_{i} \oplus y_{i} \oplus x_{i}\right]$. On the other hand, $((x \circ x) \circ y) \circ x=\left(\left(\left(x_{i}\right) \circ\left(x_{i}\right)\right) \circ\left[y_{i}\right]\right) \circ\left(x_{i}\right)=\left(\left(z_{i}\right) \circ\left[y_{i}\right]\right) \circ\left(x_{i}\right)=\left[z_{i} \oplus y_{i}\right] \circ\left(x_{i}\right)=$ $\left[z_{i} \oplus y_{i} \oplus x_{i}\right]$.

Assume that $x \in B$ and $y \in A$. Then $(x \circ x) \circ(y \circ x)=\left[x_{i}^{\prime}\right] \circ\left[y_{i} \oplus x_{i}\right]$. Note that $x_{i}^{\prime}=y_{i} \oplus x_{i}$ for every $i$ if and only if $y_{i}=1$ for every $i$. But this cannot happen since $y \in A$. Thus $\left[x_{i}^{\prime}\right] \circ\left[y_{i} \oplus x_{i}\right]=\left(x_{i}^{\prime} \oplus y_{i} \oplus x_{i}\right)=\left(y_{i}\right)$. On the other hand, $((x \circ x) \circ y) \circ x=\left(\left[x_{i}^{\prime}\right] \circ\left(y_{i}\right)\right) \circ\left[x_{i}\right]=\left[x_{i}^{\prime} \oplus y_{i}\right] \circ\left[x_{i}\right]$. For the same reason as above we cannot have $x_{i}^{\prime} \oplus y_{i}=x_{i}$ for every $i$, and thus $\left[x_{i}^{\prime} \oplus y_{i}\right] \circ\left[x_{i}\right]=\left(y_{i}\right)$.

Finally assume that $x \in B$ and $y \in B$. Then $(x \circ x) \circ(y \circ x)$ is equal to

$$
\left[x_{i}^{\prime}\right] \circ\left(\left[y_{i}\right] \circ\left[x_{i}\right]\right)= \begin{cases}{\left[x_{i}^{\prime}\right] \circ\left(y_{i} \oplus x_{i}\right)=\left[y_{i}\right],} & \text { if }\left[x_{i}\right] \neq\left[y_{i}\right], \\ {\left[x_{i}^{\prime}\right] \circ\left[x_{i}^{\prime}\right]=\left[x_{i}^{\prime \prime}\right]=\left[x_{i}\right]=\left[y_{i}\right],} & \text { otherwise }\end{cases}
$$


On the other hand, $((x \circ x) \circ y) \circ x$ is equal to

$$
\left(\left[x_{i}^{\prime}\right] \circ\left[y_{i}\right]\right) \circ\left[x_{i}\right]= \begin{cases}\left(x_{i}^{\prime} \oplus y_{i}\right) \circ\left[x_{i}\right]=\left[y_{i}\right], & \text { if }\left[x_{i}^{\prime}\right] \neq\left[y_{i}\right], \\ {\left[x_{i}^{\prime \prime}\right] \circ\left[x_{i}\right]=\left[x_{i}\right] \circ\left[x_{i}\right]=\left[x_{i}^{\prime}\right]=\left[y_{i}\right],} & \text { otherwise }\end{cases}
$$

This finishes the proof.

Lemma 6.3. Let $A$ be a commutative loop. Then every element of $\mathcal{J}(A) \backslash A$ has order 3 . In particular, when $A$ is power associative then so is $\mathcal{J}(A)$.

Proof. We must show that $(x \circ x) \circ x=x \circ(x \circ x)=(0)$ for every $x=\left[x_{i}\right] \in \mathcal{J}(A) \backslash A$. We have $(x \circ x) \circ x=\left[x_{i}^{\prime}\right] \circ\left[x_{i}\right]=\left(\left(x_{i}^{\prime} \oplus x_{i}\right)^{\prime}\right)=\left(1^{\prime}\right)=(0)$, and similarly for $x \circ(x \circ x)$.

Lemma 6.4. Let $A$ be a commutative loop of order $2^{n}-1>1$. Then $\mathcal{J}(A)$ is not left alternative, and hence not diassociative.

Proof. The commutative loop $A$ cannot be of exponent 2 by Corollary 2.4. Let $x=\left(x_{i}\right) \in A$ be an element satisfying $x^{2} \neq 1$. Let $y=[0] \in B$. Then $x \circ(x \circ$ $y)=\left(x_{i}\right) \circ\left[x_{i}\right]=[0]$. Now, $\left(z_{i}\right)=x \circ x$ is a nonidentity element of $A$, and thus $(x \circ x) \circ y=\left[z_{i}\right] \neq[0]$.

If $Q$ is a loop and $x \in Q$, denote by $L_{x}, R_{x}$ the left and right translations by $x$ in $Q$. Recall the left inner mappings $L(x, y)=L_{y x}^{-1} L_{y} L_{x}$, the right inner mappings $R(x, y)=R_{x y}^{-1} R_{y} R_{x}$, and the middle inner mappings, or conjugations $T(x)=R_{x}^{-1} L_{x}$.

A subloop $S$ of a loop $Q$ is normal, $S \unlhd Q$, if $S$ is invariant under all inner mappings of $Q$. A loop $Q$ is simple if it has no normal subloops except for $\{1\}$ and $Q$.

Proposition 6.5. Let $A$ be a simple commutative loop. Then $\mathcal{J}(A)$ is simple as well.

Proof. The statement holds when $|A|=1$, so suppose that $|A|=2^{n}-1>1$. Let $S$ be a nontrivial normal subloop of $\mathcal{J}(A)=A \cup B$. Then $S \cap A$ is a normal subloop of $A$. Since $A$ is simple, we have $S \cap A=1$ or $S \cap A=A$. The latter case implies that $|S| \geq 2^{n}-1$. Since $|S|$ has to be a divisor of $|\mathcal{J}(A)|=2^{n+1}-1$, it follows that $S=\mathcal{J}(A)$, a contradiction.

So suppose that $S \cap A=1$, and let $x \in S \backslash A$. By Lemma 6.3. $|x|=3$.

If $S$ contains $\langle x\rangle$ properly, let $y \in S \backslash(A \cup\langle x\rangle)$. Then $x \circ y=\left[x_{i}\right] \circ\left[y_{i}\right]=\left(\left(x_{i} \oplus y_{i}\right)^{\prime}\right)$. Note that $\left[y_{i}\right]=y \neq x^{2}=\left[x_{i}^{\prime}\right]$, and thus $x \circ y=\left(\left(x_{i} \oplus y_{i}\right)^{\prime}\right) \neq(0)$, contradicting $S \cap A=1$.

We can therefore assume that $S=\langle x\rangle$ for some $x \in S \backslash A$. Recall that $S$ is closed under all left inner mappings of $\mathcal{J}(A)$. Thus for every $u, v \in \mathcal{J}(A)$ there must be $z \in S$ such that $u \circ(v \circ x)=(u \circ v) \circ z$. We show that this is not the case.

First assume that $x \neq[0]$ and $x \neq[1]$. Then $[0] \circ\left([0] \circ\left[x_{i}\right]\right)=[0] \circ\left(x_{i}^{\prime}\right)=\left[x_{i}^{\prime}\right]$. But $[0] \circ[0]=[1] \neq\left[x_{i}^{\prime}\right],([0] \circ[0]) \circ\left[x_{i}\right]=[1] \circ\left[x_{i}\right]=\left(x_{i}\right) \neq\left[x_{i}^{\prime}\right]$, and $([0] \circ[0]) \circ\left(\left[x_{i}\right] \circ\left[x_{i}\right]\right)=$ $[1] \circ\left[x_{i}^{\prime}\right]=\left(x_{i}^{\prime}\right) \neq\left[x_{i}^{\prime}\right]$. Now assume that $x=[0]$, so $x^{2}=[1]$. Pick $y \in A$ such that $y^{2} \neq 1$. Then $\left(y_{i}\right) \circ\left(\left(y_{i}\right) \circ[0]\right)=\left(y_{i}\right) \circ\left[y_{i}\right]=[0]$, but $\left(y_{i}\right) \circ\left(y_{i}\right)=\left(z_{i}\right) \neq[0]$, $\left(\left(y_{i}\right) \circ\left(y_{i}\right)\right) \circ[0]=\left(z_{i}\right) \circ[0]=\left[z_{i}\right] \neq[0]$, and $\left(\left(y_{i}\right) \circ\left(y_{i}\right)\right) \circ[1]=\left(z_{i}\right) \circ[1]=\left[z_{i}^{\prime}\right]$. Note that $\left[z_{i}^{\prime}\right] \neq[0]$, else $\left[z_{i}\right]=[1],\left(z_{i}\right)=(1)$, a contradiction with $\left(z_{i}\right) \in A$. The remaining case $x=[1]$ is analogous. 
An infinite family of nonassociative simple Jordan loops is now at hand. Let $A_{0}$ be the trivial group, and $A_{i+1}=\mathcal{J}\left(A_{i}\right)$ for every $i \geq 0$. By Proposition 6.2, every $A_{i}$ is a Jordan loop. By Proposition 6.5, every $A_{i}$ is simple. By Lemma 6.4, $A_{i}$ is nonassociative for every $i \geq 2$.

Our results also imply that $\mathcal{J}\left(\mathbb{Z}_{p}\right)$ is a nonassociative simple Jordan loop whenever $p>2$ is a Mersenne prime.

\section{AcKnowledgment}

We thank the referees for several helpful comments, including the succinct algebraic description given in the proof of Lemma 2.6.

\section{REFERENCES}

[1] R. H. Bruck, Some results in the theory of linear non-associative algebras, Trans. Amer. Math. Soc. 56 (1944), 141-199.

[2] R. H. Bruck, A Survey of Binary Systems, Ergebnisse der Mathematik und Ihrer Grenzgebiete, New Series, Volume 20, Springer, 1958.

[3] O. Chein, H. O. Pflugfelder and J. D. H. Smith (eds.), Quasigroups and Loops: Theory and Applications, Sigma Series in Pure Mathematics 8, Heldermann, 1990.

[4] C. J. Colbourn and A. Rosa, Triple systems, Oxford Mathematical Monographs, Oxford Science Publications, Clarendon Press, Oxford 1999.

[5] T. Foguel, Amalgam of a loop over an idempotent quasigroup, Quasigroups and Related Systems 13 (2005), 99-104.

[6] E. G. Goodaire and R. G. Keeping, Jordan loops and loop rings, preprint.

[7] T. P. Kirkman, On a problem in combinations, Cambridge and Dublin Math. J. 2 (1897), 191-204.

[8] K. McCrimmon, A Taste of Jordan Algebras, Universitext, Springer, 2004.

[9] W. W. McCune, Mace4 Reference Manual and Guide, Tech. Memo ANL/MCS-TM-264, Mathematics and Computer Science Division, Argonne National Laboratory, Argonne, IL, August 2003. http://www.cs.unm.edu/ mccune/mace4/

[10] H. O. Pflugfelder, Quasigroups and Loops: Introduction, Sigma Series in Pure Mathematics 7, Heldermann Verlag, 1990.

[11] K. Pula, Powers of elements in Jordan loops, Commentationes Mathematicae Universitatis Carolinae, to appear.

E-mail address, Kinyon: mkinyon@math.du.edu

E-mail address, Pula: jpula@math.du.edu

E-mail address, Vojtěchovský: petr@math.du.edu

Department of Mathematics, University of Denver, 2360 S Gaylord St, Denver, CO 80208, U.S.A. 